\title{
Attaining the rate-independent limit of a rate-dependent strain gradient plasticity theory
}

El-Naaman, Salim Abdallah; Nielsen, Kim Lau; Niordson, Christian Frithiof

\section{Published in:}

Extreme Mechanics Letters

Link to article, DOI:

10.1016/j.eml.2016.04.012

Publication date:

2016

Document Version

Peer reviewed version

Link back to DTU Orbit

Citation (APA):

El-Naaman, S. A., Nielsen, K. L., \& Niordson, C. F. (2016). Attaining the rate-independent limit of a ratedependent strain gradient plasticity theory. Extreme Mechanics Letters, 9(Part 1), 40-44.

https://doi.org/10.1016/j.eml.2016.04.012

\section{General rights}

Copyright and moral rights for the publications made accessible in the public portal are retained by the authors and/or other copyright owners and it is a condition of accessing publications that users recognise and abide by the legal requirements associated with these rights.

- Users may download and print one copy of any publication from the public portal for the purpose of private study or research.

- You may not further distribute the material or use it for any profit-making activity or commercial gain

- You may freely distribute the URL identifying the publication in the public portal 


\title{
Attaining the rate-independent limit of a rate-dependent strain gradient plasticity theory
}

\author{
S.A. El-Naaman*, K.L. Nielsen, C.F. Niordson \\ Department of Mechanical Engineering, Solid Mechanics, Technical University of Denmark, \\ DK-2800 Kgs. Lyngby, Denmark
}

\begin{abstract}
The existence of characteristic strain rates in rate-dependent material models, corresponding to rate-independent model behavior, is studied within a back stress based rate-dependent higher order strain gradient crystal plasticity model. Such characteristic rates have recently been observed for steady-state processes, and the present study aims to demonstrate that the observations in fact unearth a more widespread phenomenon. In this work, two newly proposed back stress formulations are adopted to account for the strain gradient effects in the single slip simple shear case, and characteristic rates for a selected quantity are identified through numerical analysis. Evidently, the concept of a characteristic rate, within the rate-dependent material models, may help unlock an otherwise inaccessible parameter space.

Keywords: Strain gradient plasticity, Size-effects, Rate-dependent theory, Crystal plasticity, Back stress formulation
\end{abstract}

\section{Introduction}

Strain gradient plasticity theories have become an established part of contemporary solid mechanics due to the increasing interest in micron and nano scale plasticity.

\footnotetext{
*Tel: +45 4525-4020, Fax: +45 4593-1475

URL: saeln@mek.dtu.dk (S.A. El-Naaman)
} 
Experiments have demonstrated that size-dependent behavior, in terms of increased hardening and/or strengthening, is associated with spatial gradients of plastic strain in ductile crystalline materials (see e.g. Fleck et al., 1994; Mu et al., 2014). The plastic strain gradients are accommodated by a portion of lattice defects, often referred to as geometrically necessary dislocations (GNDs), which leads to a long range internal stress field. Many gradient theories of plasticity have been proposed to capture size-effects, and although so-called lower order theories have been explored (e.g. Bassani, 2001; Huang et al., 2004; Niordson and Hutchinson, 2003), the common approach involves theories of a higher order nature, which enable micro-structural boundary conditions (e.g. Fleck et al., 1994; Gurtin, 2000, 2002; Fleck and Hutchinson, 2001; Yefimov et al., 2004; Evers et al., 2004; Bayley et al., 2006; Fleck and Willis, 2009a,b). The present study employs the rate-dependent non-work conjugate type (or back stress based) higher order theory formulated by Kuroda and Tvergaard $(2006,2008)$. In this type of theory the virtual work principle remains the conventional one, while the evolution of GND densities is accounted for through additional differential equations. Here, a back stress, representing the long range internal stresses due to pile-up of GNDs, affects the plastic slip rate as kinematic hardening.

In the following study the existence of a characteristic slip rate, at which a specific macroscopic quantity becomes independent of the rate sensitivity exponent, will be demonstrated through numerical analysis of the idealized simple shear case for a single crystal. The adopted methodology represents a promising tool for obtaining rate-independent results using rate-dependent frameworks, and the extent of the matter remains to be explored. The idea of a characteristic rate was first discussed in detail 
by Nielsen and Niordson (2012) in relation to conventional rate-dependent steady-state modeling and later exploited in Nielsen et al. (2012) to extract rate-independent results from a scale-dependent steady-state framework. Nielsen (2015) also found similar results for steady-state sheet rolling. Characteristic rates may exist for a wide range of other structural problems, and a broader sense of the phenomenon is demonstrated through the results of the present study.

The paper is structured as follows. The strain gradient plasticity model and adopted back stress formulations are briefly outlined in Section 2, (details can be found in Kuroda and Tvergaard, 2006, 2008; El-Naaman et al., 2016b,a). The boundary value problem treated is described in Section 3, after which, a series of numerical results are presented in Section 4. The study is concluded in Section 5.

\section{Strain gradient crystal plasticity model}

The present study employs the strain gradient crystal plasticity theory proposed by Kuroda and Tvergaard $(2006,2008)$ within a conventional rate-dependent small strain elasto-viscoplastic framework. Hence, the total strain rate is given by; $\dot{\varepsilon}_{i j}=\left(\dot{u}_{i, j}+\dot{u}_{j, i}\right) / 2$, which is additively decomposed into an elastic part, $\dot{\varepsilon}_{i j}^{e}$, and a plastic part, $\dot{\varepsilon}_{i j}^{p}$, so that $\dot{\varepsilon}_{i j}=\dot{\varepsilon}_{i j}^{e}+\dot{\varepsilon}_{i j}^{p}$. Plastic deformation occurs as a result of crystallographic slip on the individual slip systems, and thus, the Cartesian components of the plastic strain rate is given in terms of the slip rate, $\dot{\gamma}$, on the $\alpha^{\prime}$ th slip system, as

$$
\dot{\varepsilon}_{i j}^{p}=\sum_{\alpha} \dot{\gamma}^{(\alpha)} P_{i j}^{(\alpha)}, \quad P_{i j}^{(\alpha)}=\frac{1}{2}\left(s_{i}^{(\alpha)} m_{j}^{(\alpha)}+m_{i}^{(\alpha)} s_{j}^{(\alpha)}\right) .
$$

The superposed dots denote material time derivative, $P_{i j}^{(\alpha)}$ is the Schmid orientation tensor, and unit vectors $s_{i}^{(\alpha)}$ and $m_{i}^{(\alpha)}$ specify the slip direction and the slip plane 
normal, respectively (see Fig. 1). The equilibrium equations for the non-work conjugate formulation are given by conventional stress equilibrium in absence of body forces; $\sigma_{i j, j}=0$, where the Cauchy stress rate tensor is given by; $\dot{\sigma}_{i j}=\mathscr{L}_{i j k l}\left(\dot{\varepsilon}_{k l}-\dot{\varepsilon}_{k l}^{p}\right)$, in which $\mathscr{L}_{i j k l}$ is the fourth order elastic stiffness tensor. Thereby, the conventional incremental principle of virtual work reads

$$
\int_{V} \mathscr{L}_{i j k l} \dot{\varepsilon}_{k l} \delta \dot{\varepsilon}_{i j} \mathrm{~d} V=\int_{V} \mathscr{L}_{i j k l} \dot{\varepsilon}_{k l}^{p} \delta \dot{\varepsilon}_{i j} \mathrm{~d} V+\int_{S} \dot{T}_{i} \delta \dot{u}_{i} \mathrm{~d} S, \quad \dot{T}_{i} \equiv \dot{\sigma}_{i j} n_{j},
$$

where $\dot{T}_{i}$ are the traction rates, $n_{i}$ is the outward unit normal to the surface $S$ bounding the volume $V$. In a two dimensional setting, an additional partial differential equation accounts for the evolution of GND density; $1 / b \gamma_{, i}^{(\alpha)} s_{i}^{(\alpha)}+\rho_{G}^{(\alpha)}=0$, where $b$ is the magnitude of the Burgers vector, and $\rho_{G}^{(\alpha)}$ is the GND density of edge type on slip system $\alpha$ (Ashby, 1970). The GND density balance equation is expressed on weak form as

$$
\frac{1}{b} \int_{V} \delta \rho_{, i} s_{i}^{(\alpha)} \gamma^{(\alpha)} \mathrm{d} V=\frac{1}{b} \int_{S} \delta \rho \zeta^{(\alpha)} \mathrm{d} S+\int_{V} \delta \rho \rho_{G}^{(\alpha)} \mathrm{d} V, \quad \zeta^{(\alpha)} \equiv \gamma^{(\alpha)} n_{i} s_{i}^{(\alpha)},
$$

where $\delta \rho$ is a weighting function (or virtual GND density) ${ }^{1}$.

A perfectly plastic, gradient-enhanced version of the widely used conventional power law slip rate relation (Hutchinson, 1976; Peirce et al., 1983) is employed, so that

$$
\dot{\gamma}^{(\alpha)}=\dot{\gamma}_{0} \operatorname{sgn}\left(\tau^{(\alpha)}-\tau_{b}^{(\alpha)}\right)\left(\frac{\left|\tau^{(\alpha)}-\tau_{b}^{(\alpha)}\right|}{\tau_{0}}\right)^{1 / m},
$$

where $\dot{\gamma}_{0}$ is a reference slip rate, $\tau^{(\alpha)}$ is the Schmid stress $\left(\tau^{(\alpha)}=\sigma_{i j} P_{i j}^{(\alpha)}\right), \tau_{b}^{(\alpha)}$ is a back stress, $m$ is the rate sensitivity exponent, and $\tau_{0}$ is the critical resolved shear stress ${ }^{2}$.

\footnotetext{
${ }^{1}$ For a detailed discussion on the micro-structural boundary conditions see Kuroda and Tvergaard (2008).

${ }^{2}$ Note that for $\tau_{b}^{(\alpha)}$ equal to zero, Eq. (4) reduces to the conventional theory.
} 
The back stress, $\tau_{b}^{(\alpha)}$, is phenomenologically related to the distribution of the GND density, and accounts for the long range internal stresses due to dislocation pile-up. In the present study, two back stress formulations, proposed in El-Naaman et al. (2016b), are adopted. One is a thermodynamically consistent formulation derived from a free energy potential

$$
\tau_{b}^{(\alpha)}=\mu \tau_{0} b^{\mu} L^{\mu+1}\left(\left|\rho_{G}^{(\alpha)}\right|+\rho_{0}\right)^{\mu-1} \rho_{G, i}^{(\alpha)} s_{i}^{(\alpha)} \quad, \quad 0<\mu \leq 1,
$$

where $\rho_{0}$ is a non-zero numerical parameter, which resembles the presence of statistically stored dislocations (see e.g. Groma et al., 2003; Yefimov et al., 2004). Note that Eq. (5) corresponds to a quadratic free energy for $\mu=1$.

The second back stress relation employed in the present study is given by the piecewise function,

$$
\tau_{b}^{(\alpha)}= \begin{cases}b \tau_{0} L^{2} \rho_{G, i}^{(\alpha)} s_{i}^{(\alpha)} & \text { for }\left|\tau_{b}^{(\alpha)}\right| \leq \tau_{T}, \\ \operatorname{sgn}\left(\rho_{G, i}^{(\alpha)} s_{i}^{(\alpha)}\right) b^{\kappa} \tau_{T}^{1-\kappa} \tau_{0}^{\kappa} L^{2 \kappa}\left|\rho_{G, i}^{(\alpha)} s_{i}^{(\alpha)}\right|^{\kappa} & , \text { for }\left|\tau_{b}^{(\alpha)}\right|>\tau_{T}\end{cases}
$$

where $0 \leq \kappa \leq 1$ is assumed and $\tau_{T}$ defines a transition point, from a quadratic free energy based back stress, into a power law dependence on the GND density gradients. Note that Eq. (6) corresponds to a quadratic free energy for $\kappa=1$, but thermodynamical consistency is not guaranteed for other values of $\kappa$. However, the numerical solutions presented, have been found to satisfy positive dissipation throughout the loading history, such that; $\sigma_{i j} \dot{\varepsilon}_{i j}^{p}=\sum_{\alpha} \tau^{(\alpha)} \dot{\gamma}^{(\alpha)} \geq 0$.

For a detailed description of the adopted back stress formulations and choice of model parameters see El-Naaman et al. (2016a,b). 


\section{Boundary value problem}

The single slip simple shear problem, with positive slip in the $x_{2}$-direction, is considered in order to demonstrate the rate-dependent behavior of the adopted back stress based strain gradient plasticity model (see Fig. 1 for a schematic illustration and definition of the slip system). The following model parameters are used throughout: Young's modulus $E=130 \mathrm{GPa}$, Poisson's ratio $\nu=0.3, \tau_{0}=50 \mathrm{MPa}$, and $b=0.286 \mathrm{~nm}$. The following two back stress model parameters are used: $\rho_{G, 0}=10^{5} \mathrm{~mm}^{-2}$ and $\tau_{T} / \tau_{0}=$ 0.06 in Eqs. (5) and (6), respectively. Periodicity in the $x_{1}$-direction is imposed for the material domain of height $H$ and width $W$, such that

$$
\begin{aligned}
& u_{i}\left(0, x_{2}\right)=u_{i}\left(W, x_{2}\right) \\
& \rho_{G}^{(\alpha)}\left(0, x_{2}\right)=\rho_{G}^{(\alpha)}\left(W, x_{2}\right) .
\end{aligned}
$$

The following additional boundary conditions are imposed on the displacement field:

$$
\begin{aligned}
& u_{1}=u_{2}=0 \text { at } x_{2}=0 \\
& u_{1}=U(t) \text { and } u_{2}=0 \text { at } x_{2}=H .
\end{aligned}
$$

The top and bottom boundaries of the material domain are microscopically passivated by the following higher order boundary conditions imposed on the GND density field equation:

$$
\zeta^{(\alpha)} \equiv \gamma^{(\alpha)} n_{i} s_{i}^{(\alpha)}=0 \text { at } x_{2}=0 \text { and } x_{2}=H \text {. }
$$

This models that dislocations are unable to exit through these boundaries.

The conventional and micro-structural equilibrium equations are solved in a staggered scheme, using the finite element method (FEM), with time integration carried out by the forward Euler method. Eight-node isoparametric quadrilateral elements, with 
reduced Gauss integration, are used for the displacement field analysis, and equivalent four-node elements, with full Gauss integration, are used for the GND density field analysis. The employed finite element mesh consists of 58 elements in the $x_{2}$-direction and includes a refined mesh at the microscopically passivated boundaries to resolve the possibly steep gradients.

\section{Methods, results, and discussion}

The present numerical study focuses on the macroscopic response of the adopted gradient crystal plasticity model, where the model rate sensitivity is investigated, and a problem dependent characteristic rate is identified for the constrained simple shear problem. The material domain is sheared to a macroscopic shear strain $\Gamma=0.03$, after which, one complete load cycle is performed, so that the macroscopic shear strain goes once to -0.03 and back to 0.03 . Care was taken to ensure that a steady state cyclic response is obtained by running multiple load cycles about the zero strain axis.

Figures 2 and 3 show the shear stress vs. macroscopic shear strain using the thermodynamically consistent back stress formulation (Eq. (5)) and the purely phenomenological back stress (Eq. (6)), respectively. Results are shown for a macroscopic shear rate of $\dot{\Gamma}=2 \dot{\gamma}_{0}$, for different values of the material length parameter, $L$, and for different values of the back stress exponents $\mu$ and $\kappa$, respectively. In both cases, the response curves for $L / H=10$ show a seemingly anomalous change in slope during plastic flow with inflection points close to zero strain. This phenomenon is discussed in detail in El-Naaman et al. (2016a) and will not be elaborated in the present paper.

In order to identify the characteristic rate for a given specific quantity, a simple procedure is carried out. This procedure implies running a series of calculations for 
different values of the rate exponent, $m$, so that the quantity of interest can be plotted as function of the applied rate for each value of $m$. The convergent intersection between the curves then reveals the quantity specific rate-independent point. The existence of a well defined intersection is contingent upon the quantity investigated being monotonically increasing or decreasing with the rate sensitivity in an interval above and below the characteristic rate, respectively. Note that in order to identify the rateindependent point it is not necessary to employ low values of $m$, since the intersection may be obtained for curves at any given rate sensitivity. Indirectly introduced through the reference slip rate, $\dot{\gamma}_{0}$, in Eq. (4), the rate-independent point, tied to the characteristic rate, may open the door for a parameter space, which is otherwise inaccessible due to the numerical difficulties associated with the limit $m \longrightarrow 0$ (see also Nielsen et al., 2012).

The Kuroda and Tvergaard $(2006,2008)$ theory is not formulated in a rate-independent framework, however, rate-independent results may readily be extracted by identifying the characteristic rate through the aforementioned procedure. In the following, this will be demonstrated for the maximum attained shear stress on the hysteresis curve, $\left|\tau_{12}\right|_{\max }$.

Figures 4 and 5 show $\left|\tau_{12}\right|_{\max }$ as function of the applied shear rate. Figure 4 shows results obtained using the thermodynamically consistent back stress formulation (Eq. (5)) for different values of $\mu$ and $L$, and Fig. 5 shows results obtained using the purely phenomenological back stress formulation (Eq. (6)) for different values of $\kappa$ and $L$. Clearly convergent intersections are observed for both back stress models. In both formulations, the intersections occur at a higher shear stress when $L$ is increased, due 
to the increased strain gradient hardening. Furthermore, the intersection translates slightly toward lower rates for increasing $L^{3}$.

Analogous to the results obtained for the maximum shear stress, $\left|\tau_{12}\right|_{\text {max }}$, rateindependent points also exist for the dissipated hysteresis energy, which is defined as the area inside the hysteresis loop. However, these results are omitted in the present paper, since a high level of coincidence between the curves impair a graphical representation. This is due to the fact that, while the shape of the hysteresis loop changes significantly, the area remains approximately the same for all computations, as one may perceive from Figs. 2 and 3. The uncertainty in the intersections for the hysteresis energy were found to be on the order of 0.5 percent (or below).

\section{Conclusion}

The existence of a characteristic rate at which specific macroscopic quantities reveal their rate-independent value has been demonstrated for the pure shear case of an infinite slab between rigid platens. Here, considering the visco-plastic higher order strain gradient crystal plasticity model proposed by Kuroda and Tvergaard (2006, 2008). Both when assuming a quadratic free energy, but also when adopting the newly proposed back stress formulations (El-Naaman et al., 2016b,a), clear and unambiguous intersections (defining the characteristic rate) are found when plotting the quantities vs. deformation rate for various rate sensitivity exponents. Specific to the purely phenomenological back stress formulation (Eq. (6)), the transition parameter $\tau_{T}$ is

\footnotetext{
${ }^{3}$ The peak in strain gradient hardening at intermediate values of $\mu$ (also reported in El-Naaman et al., 2016b) is evident in Figs. 2 and 4 a for $\mu=0.3$.
} 
significant for the quality of the intersection and should be chosen carefully. Choosing $\tau_{T}$ too high can result in a response dominated by quadratic free energy. In general, the choice of back stress models was found to have a slight influence on the resulting characteristic rate as well as the corresponding rate-independent value of the objective quantity.

The characteristic rate is strongly tied to the characteristic time introduced into the material model by the reference strain rate, $\dot{\gamma}_{0}$, and is fundamental to the power law type strain rate hardening relations used in the visco-plastic theories. The presented results and observations establish the findings reported in Nielsen and Niordson (2012), Nielsen et al. (2012) and Nielsen (2015) as a widespread phenomenon, and show that by exploiting it, results at the rate-independent limit may, in fact, be reached for the present model, which only exists in a rate-dependent framework. It should be made clear, however, that the phenomenon is a purely theoretical concept and, to the best of the authors knowledge, remains to be observed in experiments. Regardless of this, the concept is highly significant in modeling contexts, since it does in fact exist within widely used visco-plastic theories. Bearing this in mind, the authors encourage experimental investigations to clarify the matter.

\section{Acknowledgments}

The work is financially supported by The Danish Council for Independent Research under the research career programme Sapere Aude as part of the project "Higher Order Theories in Solid Mechanics", grant 11-105098/FTP. 


\section{References}

Ashby, M., 1970. The deformation of plastically non-homogeneous alloys. Philos. Mag. 21, 399-424.

Bassani, J., 2001. Incompressibility and a simple gradient theory of plasticity. Journal of the Mechanics and Physics of Solids 49, 1983-1996.

Bayley, C., Brekelmans, W., Geers, M., 2006. A comparison of dislocation induced back stress formulations in strain gradient crystal plasticty. International Journal of Solids and Structures 43, 7268-7286.

El-Naaman, S., Nielsen, K., Niordson, C., 2016a. An investigation of back stress formulations under cyclic loading, Submitted.

El-Naaman, S., Nielsen, K., Niordson, C., 2016b. On modeling micro-structural evolution using a higher order strain gradient continuum theory. International Journal of Plasticity 76, 285-298.

Evers, L., Brekelmans, W., Geers, M., 2004. Non-local cystal plasticity model with intrinsic SSD and GND effects. Journal of the Mechanics and Physics of Solids 52, 2379-2401.

Fleck, N., Hutchinson, J., 2001. A reformulation of strain gradient plasticity. Journal of the Mechanics and Physics of Solids 49, 2245-2271.

Fleck, N., Muller, G., Ashby, M., Hutchinson, J., 1994. Strain gradient plasticity: Theory and experiment. Acta Metallurgica et materialia 42 (2), 475-487. 
Fleck, N., Willis, J., 2009a. A mathematical basis for strain-gradient plasticity theory. Part I: Scalar plastic multiplier. Journal of the Mechanics and Physics of Solids 57, $161-177$.

Fleck, N., Willis, J., 2009b. A mathematical basis for strain-gradient plasticity theory. Part II: Tensorial plastic multiplier. Journal of the Mechanics and Physics of Solids $57,1045-1057$.

Groma, I., Csikor, F., Zaizer, M., 2003. Spatial correlations and higher-order gradient terms in a continuum description of dislocation dynamics. Acta Materialia 51, 12711281.

Gurtin, M., 2000. On the plasticity of single crystals: free energy, microforces, plasticstrain gradients. Journal of the Mechanics and Physics of Solids 48 (5), 989-1036.

Gurtin, M., 2002. A gradient theory of single-crystal viscoplasticity that accounts for geometrically necessary dislocations. Journal of the Mechanics and Physics of Solids $50,5-32$.

Huang, Y., Qu, S., Hwang, K., Li, M., Gao, H., 2004. A conventional theory of mechanism-based strain gradient plasticity. International Journal of Plasticity 20, $753-782$.

Hutchinson, J., 1976. Bounds and self-consistent estimates for creep of polycrystalline materials. Proc. R. Soc. Lond. A 348, 101-127.

Kuroda, M., Tvergaard, V., 2006. Studies of scale dependent crystal viscoplasticity models. Journal of the Mechanics and Physics of Solids 54, 1789-1810. 
Kuroda, M., Tvergaard, V., 2008. On the formulation of higher-order strain gradient crystal plasticity models. Journal of the Mechanics and Physics of Solids 56, 15911608.

Mu, Y., Hutchinson, J., Meng, W., 2014. Micro-pillar measurements of plasticity in confined cu thin films. Extreme Mechanics Letters 1, 62-69.

Nielsen, K., 2015. Rolling induced size effects in elastic-viscoplastic sheet metals. European Journal of Mechanics A/Solids 53, 259-267.

Nielsen, K., Niordson, C., 2012. Rate sensitivity of mixed mode interface toughness of dissimilar meallic materials: Studied at steady state. International Journal of Solids and Structures 49, 576-583.

Nielsen, K., Niordson, C., Hutchinson, J., 2012. Strain gradient effects on steady state crack growth in rate-sensitive materials. Engineering Fracture Mechanics 96, 61-71.

Niordson, C., Hutchinson, J., 2003. On lower order strain gradient plasticity theories. European Journal of Mechanics A/Solids 22, 771-778.

Peirce, D., Asaro, R., Needleman, A., 1983. Material rate dependence and localized deformation in crystalline solids. Acta Metall. 31, 1951-1976.

Yefimov, S., Groma, I., van der Giessen, E., 2004. A comparison of a statisticalmechanics based plasticity model with descrete dislocation plasticity calculations. Journal of the Mechanics and Physics of Solids 52, 279-300. 


\section{Figures}

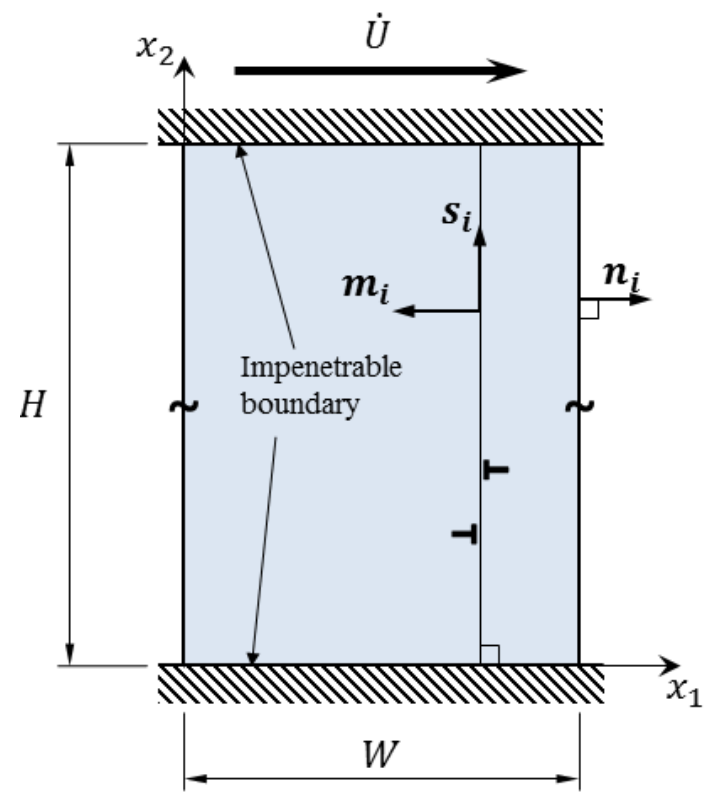

Figure 1: Schematic of the simple shear problem showing a thin film of height $H$ between rigid platens, modeled as infinite in the $x_{1}$-direction with model domain width $W$. The film has a single vertical slip system, which is passivated at the top and bottom horizontal boundaries. The domain is subject to a prescribed displacement rate $\dot{U}$.

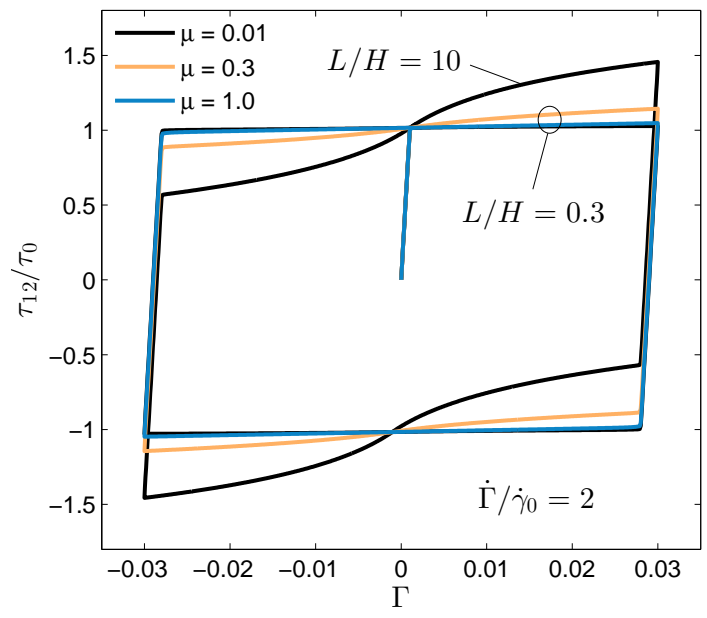

Figure 2: Numerical results for the single slip simple shear problem (Fig. 1) subject to one hysteretic cycle at a macroscopic shear rate of $\dot{\Gamma}=2 \dot{\gamma}_{0}$, using the thermodynamically consistent back stress (Eq. (5)). Results are shown for different values of $\mu$ and $L$, with $m=$ 0.02 and $\rho_{0}=10^{5} \mathrm{~mm}^{-2}$. 


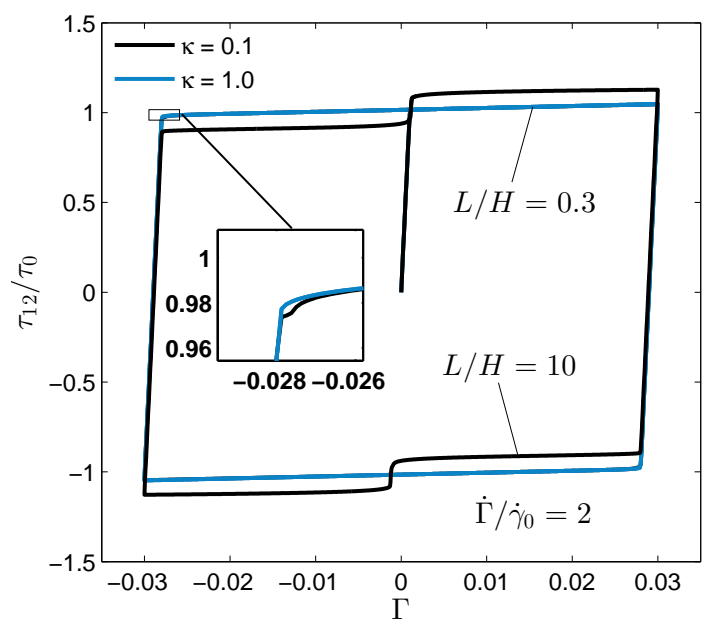

Figure 3: Numerical results for the single slip simple shear problem (Fig. 1) subject to one hysteretic cycle at a macroscopic shear rate of $\dot{\Gamma}=2 \dot{\gamma}_{0}$, using the purely phenomenologically devised back stress (Eq. (6)). Results are shown for different values of $\kappa$ and $L$, with $m=$ 0.02 and $\tau_{T} / \tau_{0}=0.06$.

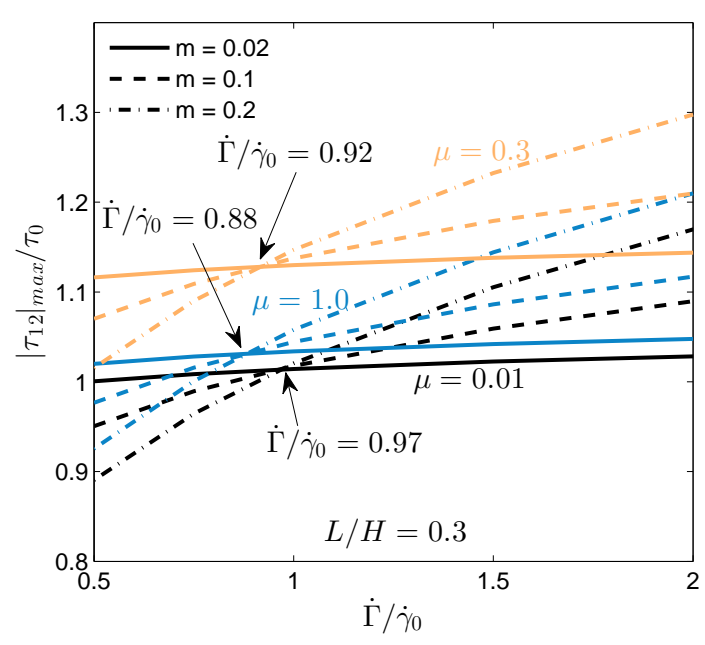

(a)

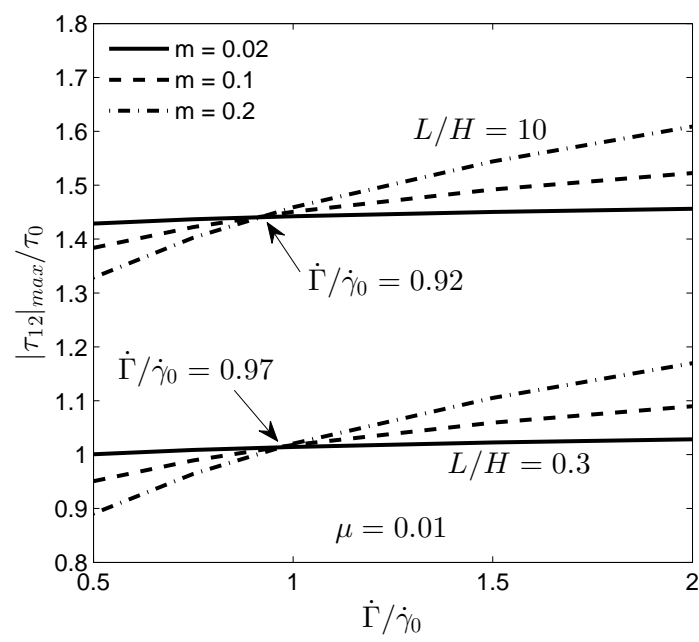

(b)

Figure 4: Maximum shear stress vs. macroscopic shear strain rate for three different values of the rate sensitivity exponent $m$. The figure shows (a) results for three different values of $\mu$ with $L / H=0.3$, and (b) results for two different values of $L$ with $\mu=0.01$. The characteristic rates corresponding to the intersections are given in the figures. 


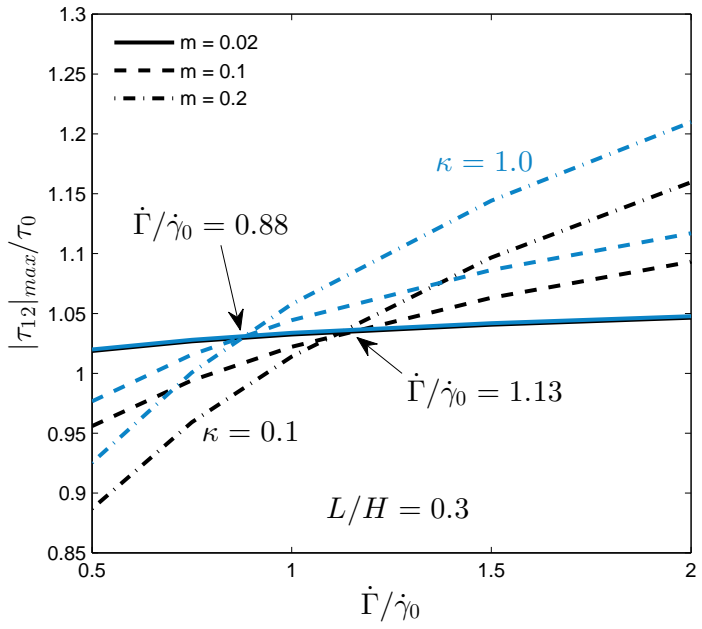

(a)

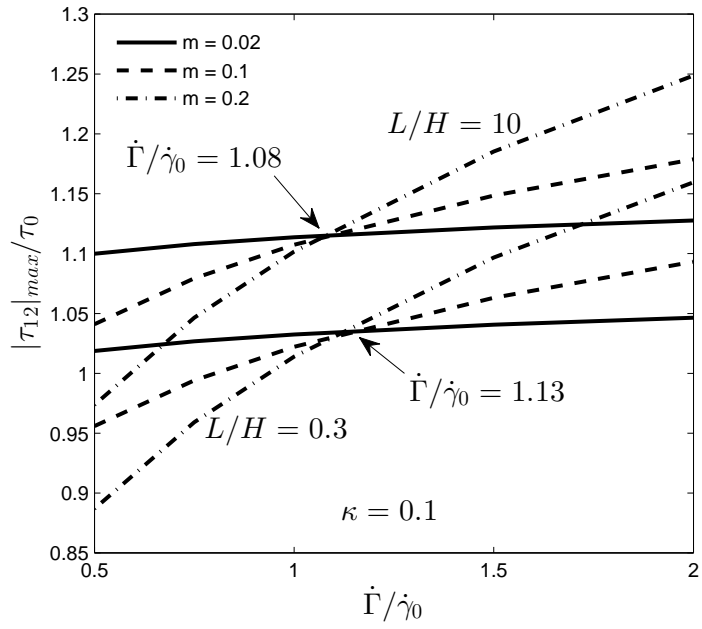

(b)

Figure 5: Maximum shear stress vs. macroscopic shear strain rate using the back stress power law (Eq. (6)) for three different values of the rate sensitivity exponent $m$. The figure shows (a) results with $\kappa=0.1$ and $\tau_{T} / \tau_{0}=0.06$, compared with the the classical quadratic gradient energy based back stress, both with $L / H=0.3$, and (b) shows results for two different values of $L$ with $\kappa=0.1$. The characteristic rates corresponding to the intersections are given in the figures. 